\title{
Magnificent marriage of scientific and artistic inquiry
}

Previously published at www.cmaj.ca

Leonardo da Vinci: The Mechanics of Man Vancouver Art Gallery

Feb. 2 to May 2, 2010

A s the 2010 Winter Olympics showcased exceptional athletes and the remarkable feats of the human body, the Vancouver Art Gallery fittingly launched Leonardo da Vinci: The Mechanics of Man. This is the first complete exhibit of Anatomical Мапиscript $A$, comprising 18 linen folio pages (many of them two-sided) with 34 pen-and-ink drawings of the structures of the body and the movements of musculature. It is a magnificent marriage of scientific and artistic inquiry.

These drawings were intended as the basis of da Vinci's treatise on human anatomy, which was never completed. Had it been otherwise, teaching in the medical schools and universities of Europe would have been much more accurate over the next 300 or more years, according to Martin Clayton, the exhibit's curator and a deputy curator of the Print Room of the Royal Collection, Windsor, England, which lent the drawings to the Vancouver Art Gallery.

These works predate Vesalius's $D e$ humani corporis fabrica by 33 years, and were drawn from human bodies legally acquired by the University of Pavia (south of Milan, Italy) and carefully dissected by da Vinci himself 30 in total over his lifetime. From initial notes, he made these drawings during the winter of $1510-11$.

This collection is remarkable for $\propto$ many reasons, not the least of which is $\approx$ the fact that the body was largely unknown and unmapped in 1510. The drawings display da Vinci's exceptional skill, in many cases a poignant combination of life study and human anatomy, such as his depictions of the musculature

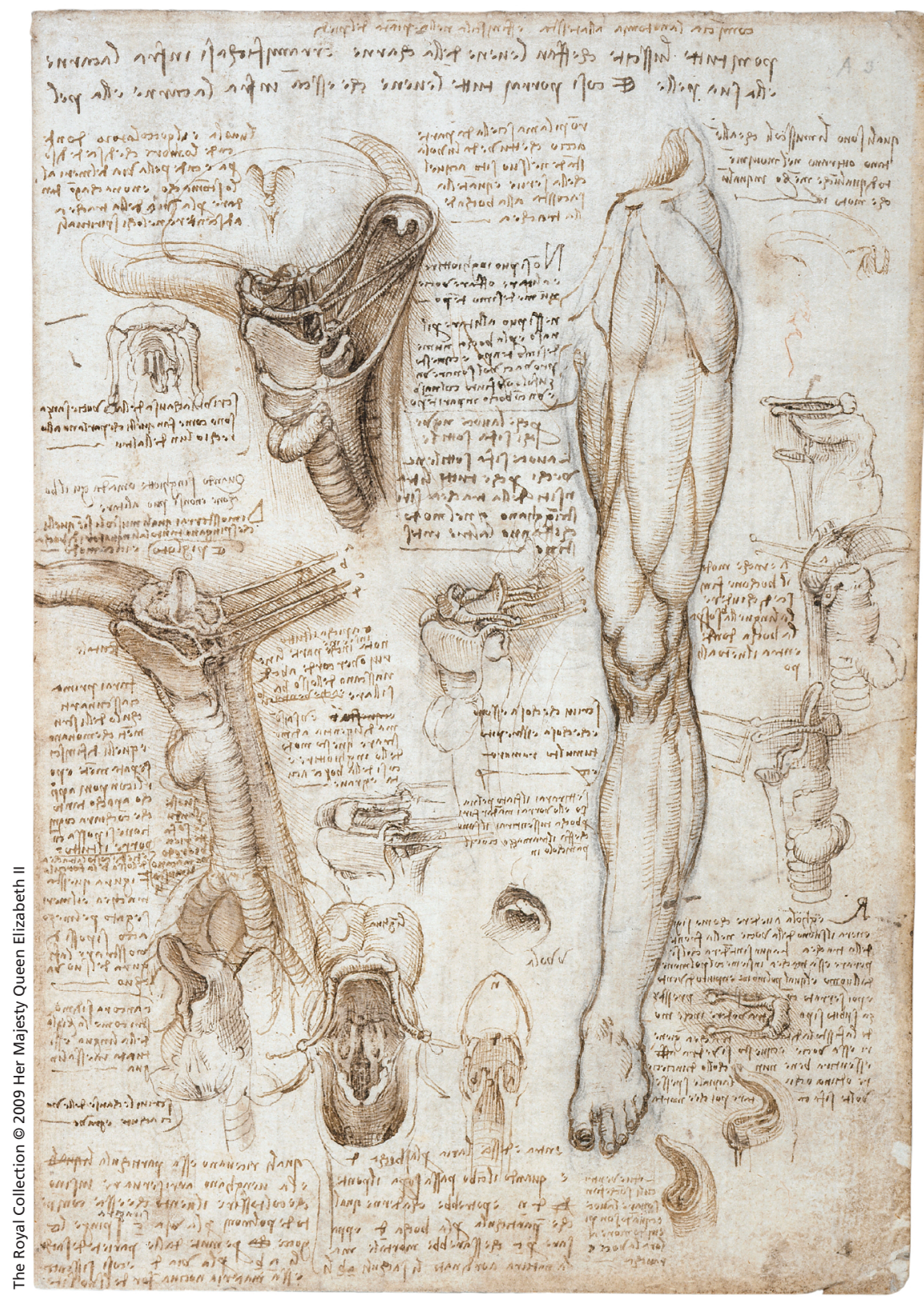

Leonardo da Vinci, The throat, and the muscles of the leg Folio 3r (1510-11). Pen and ink with wash, over traces of black chalk; red chalk sketch at top right. $29 \mathrm{~cm} \times 19.6 \mathrm{~cm}$.

of the shoulder and arm. But most striking are the complementary components of each folio page: 16 of the 18 feature multiple sketches from various perspec- tives, all surrounded by text. Some of da Vinci's notes explain, for example, the physics of toe movement. Some are a form of diary or reminder: "Use the same 
rule for the foot that you used for the hand, that is depicting first the bones from six aspects, from behind, in front, below, above, inside and outside." Others are imperative: "Provide a reason why nature has varied the five upper vertebrae of the neck at their point." Still others are about finances: "that avarice does not constrain you to make the prints in [woodcut]."

The pages, intended or not, are well designed, encouraging the eye to move and the brain to think. On folio $8 \mathrm{~V}$, between two views of the spine is da Vinci's quick explanation that the seven vertebrae of the neck sheath nerves that "spread through the arms, giving them feeling." In the lower left corner, what appears to be an exploded view of a set of gears upon closer examination reveals the first three vertebrae of the neck, separated to show their individual details. This was likely the first use of exploded perspective in Western art. Immediately beneath is this claim: "Thus you will give true knowledge of their shapes, which is impossible for either ancient or modern writers." On that one page are five drawings and 24 small blocks of text in da Vinci's well-known mirror writing.

Almost all the pages contain such pastiches of sketches, information,

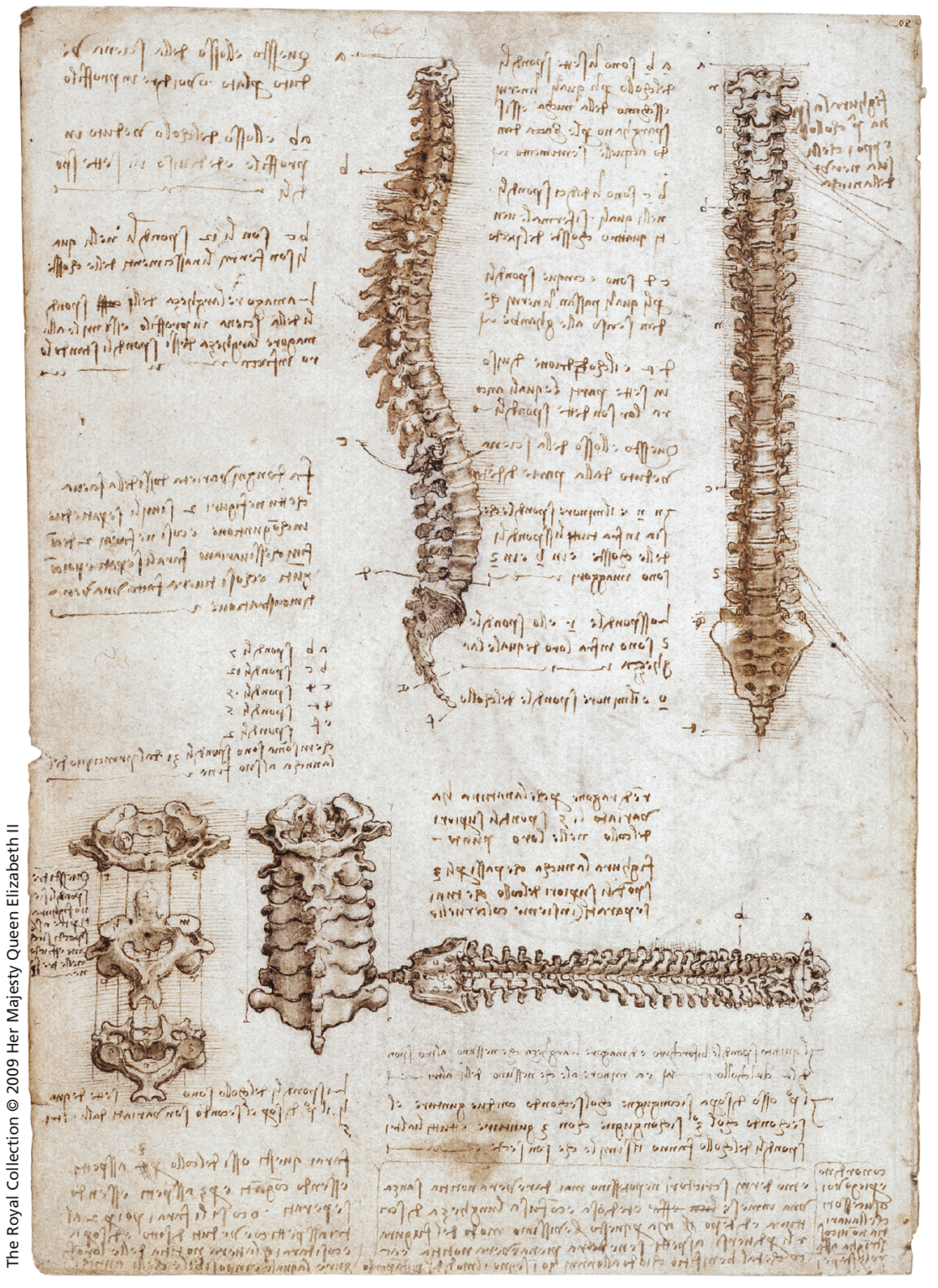

Leonardo da Vinci, The vertebral column Folio $8 \mathrm{~V}$ (1510-11). Pen and ink with wash, over traces of black chalk. $28.6 \mathrm{~cm} \times 20 \mathrm{~cm}$. questions, speculation and explanation. Each is an intimate work-in-progress; the viewer senses the passion, curiosity, and unrelenting intelligence of this artist and scientist. Taken together, the pages are in modern terms a documentary of a gifted man breaking revolutionary ground in anatomical history.

After studying the folio pages, another striking element comes clear. Despite his insistence that his images will reveal what language cannot, da Vinci's texts grow longer and more elaborate as the folio progresses. Not satisfied with visual precision, da Vinci explains why our bodies move, how we are able to pivot, bend, flex, turn our palms up, or breathe when we bend over. Under da Vinci's hand, these bodies are far from inert, and the viewer quickly learns that our anatomy has built-in redundancies that allow some muscles to compensate for others that are injured, that the human structure attains balance and equilibrium through its architecture. His numerous aspects of extended limbs, turned heads and rotated wrists allow the viewer to imagine the body in motion and also to understand that our intricate rigging of skin, vein, tendon, muscle, nerve and bone, relies on fundamental yet sophisticated physics to make us the agile creatures we are.

We must thank da Vinci's passion for detail for these remarkable insights. His dissections carefully descended from the surface of the body to its innermost recesses. He even reproduces the larynx and explains why the voice becomes weak in the elderly, as well as showing, in folio $13 \mathrm{~V}$, the mechanics of our facial expressions through muscles that are extremely hard to reveal in dissection because they attach to deep surfaces of the skin. As well, to make interior details clear to the viewer, da Vinci separates the elements in question, so each could be accurately rendered and readily comprehended.

The Olympics was a stage for exceptional human performance. The Mechanics of Man shows, however, that the mechanics of the human body itself is exceptional.

\section{Bob Sherrin BFA MFA}

Artist and author

Vancouver, BC 